\title{
Reported UAV incidents in Canada: analysis and potential solutions
}

\author{
Paul R. Nesbit ${ }^{1 *}$, Thomas E. Barchyn ${ }^{1}$, Chris H. Hugenholtz ${ }^{1}$, Sterling Cripps ${ }^{2}$, Maja Kucharczyk ${ }^{1}$ \\ ${ }^{1}$ Department of Geography, University of Calgary, 2500 University Drive NW, Calgary, AB, T2N 1N4, \\ Canada \\ ${ }^{2}$ Canadian Unmanned Incorporated, P.O. Box 21031, Medicine Hat, AB, T1A 8M3, Canada \\ *corresponding author: paul.nesbit@ucalgary.ca
}

\begin{abstract}
UAV incidents were analyzed using data from Transport Canada's Civil Aviation Daily Occurrence Reporting System (CADORS). Between 05 November 2005 and 31 December 2016 a total of 355 incidents were reported in Canadian airspace. The largest number involved UAV sightings $(66.5 \%)$ and close encounters with piloted aircraft (22.3\%). These incidents increased markedly after 2013, with the highest number in British Columbia, followed by Ontario, Quebec, Alberta, and Manitoba. The vast majority of UAV incident reports were filed by pilots of piloted aircraft. Typically, airspace at altitudes greater than 400 feet above ground level (AGL) is off limits to UAVs; however, of the 270 incidents in the CADORS database with UAV altitude reported, $80.4 \%$ were above 400 feet AGL and $62.6 \%$ were above 1000 feet AGL. Of the 268 incidents with reported horizontal distance to the nearest aerodrome, $74.6 \%$ occurred or likely occurred within 5 nautical miles $(\mathrm{nm})$, and of those $92.4 \%$ and $76.6 \%$ were reported above 100 and 300 feet AGL, respectively. Collectively, the CADORS data indicate that the overwhelming majority of UAV incidents reported in Canada were airspace violations. These results can guide future risk mitigation measures, hardware/software solutions, and educational campaigns to increase airspace safety.
\end{abstract}

Publisher's version of this article: Nesbit, P.R., Barchyn, T.E., Hugenholtz, C.H., Cripps, S. and Kucharczyk, M., 2017. Reported UAV incidents in Canada: analysis and potential solutions. Journal of Unmanned Vehicle Systems, 5(1), pp.1-11. doi: 10.1139/juvs-2016-0033

Key words: UAV, unmanned aerial vehicle, drone, aviation safety, regulation, incident 


\section{Introduction}

The flooding of global airspace with millions of UAVs has created new challenges for regulators (Gettinger and Michel, 2015). Successfully integrating this unprecedented influx of new aircraft with existing airspace regulations, while ensuring safety of both the public and other airspace users, is difficult. While it has not yet occurred or been reported, there is concern of a UAV grounding a large aircraft (Gettinger and Michel, 2015). The popularity of small UAVs in research, recreation, civil, and commercial applications is likely to continue to grow; therefore, understanding the details of typical incidents involving small UAVs is a component of the process to build better regulations and increase safety within Canadian airspace.

Although UAVs differ widely in capabilities and form, from fixed wing (Figure 1a) to rotary wing (Figure 1b), all UAVs have a number of features that make them fundamentally different than piloted aircraft.

First, most small UAVs $(<25 \mathrm{~kg})$ are far easier to operate than other aircraft. This is the result of widely available miniaturized sensor, computing, and communications technology developed for consumer smartphones. This hardware, combined with advanced flight control algorithms, has allowed simple, automated flight control. This differs from conventional radio controlled (RC) model aircraft, where the operator must manually control the aircraft.

Second, most UAVs are completely computer-controlled during flight. Having commands from either the user (through a flight controller) or a flight navigation program interpreted by a computer implies that the flight computer onboard the UAV is fully aware of its location and other relevant flight parameters. This means user commands can be evaluated by the computer, and flagged if unsafe or in violation of airspace regulations. This characteristic differs substantially from piloted aircraft, where protocols and flight control structures were developed in an era where computers were not relied upon as much as they are today, and the risk of allowing full computer control is considered too large. Full computer control is widely understood to result in less pilot engagement and short notice piloting capacity. This problem is presently a major concern in self-driving cars (a similar human/robot interface), where full 'pilot' re-engagement in an unexpected event is difficult without a delay (Merat et al., 2014).

Third, due to their relative ease of use, small UAVs can be controlled by operators with limited skill and training. Many UAV manufacturers advertise that no flying skills are required. While UAV applications were historically dominated by government and military agencies (Hugenholtz et al., 2012; Joslin, 2015), there are now a wide array of both established and emerging civil and commercial applications (e.g., Whitehead et al., 2014; Hugenholtz et al., 2015; Tamminga et al., 2015), in addition to recreational uses. Increasingly, small UAVs are being used to accomplish a task (e.g., photography, surveying, etc.). Consequently, UAV operators may be different from many other aviators (Clothier et al., 2015).

Fourth, small UAV crashes are generally low consequence. Unlike piloted aircraft, UAV operators are typically not injured in aircraft groundings, and there is no 'shared fate' when problems occur. Small UAVs have recently become inexpensive such that occasional crashes are accepted with limited concern. Furthermore, if operating legally, UAV accidents are more likely to occur in areas where there is low potential for injury or property damage (away from people, aerodromes, and infrastructure). In general, small UAVs are perceived as low risk technology (Clothier et al., 2015). However, large consequence incidents are possible (Gettinger and Michel, 2015) and reported occasionally (e.g., http://www.bbc.com/news/uk-england-hereford-worcester-34936739 [accessed 28 January 2017]).

And finally, UAV operators are remote, often far away from the aircraft. In the case of unsafe operation, finding the UAV operator can be difficult. Furthermore, UAVs can be operated over the internet, making it effectively impossibly to locate the human operator. This characteristic of anonymity and physical dissociation makes prosecution in the case of unsafe behaviour inefficient for authorities (Clarke and Moses, 2014). 
Collectively, these characteristics make UAVs different from piloted aircraft: UAVs are likely to suffer from different problems and require different solutions. Previous efforts to understand UAV safety have focused primarily on military reports from the United States (e.g., Manning et al., 2004) or quantitatively estimating risks from a probability perspective (e.g., Stevenson et al., 2015). Gettinger and Michel (2015) examined UAV incidents consisting of 'sightings' and 'close encounters' in the US National Airspace System and found 921 occurrences over a 21-month period from 2013 to 2015. Here, we expand Gettinger and Michel's (2015) analysis, examining the situation in Canada up to the end of 2016. We discuss potential solutions, both regulatory and technological for small UAVs, which could improve safety for both other airspace users and the public.

\section{Data collection and results}

\subsection{Overview of CADORS data}

The data used in this study were extracted from the Transport Canada Civil Aviation Daily Occurrence Reporting System (CADORS [available at http://wwwapps.tc.gc.ca/Saf-Sec-Sur/2/CADORSSCREAQ/m.aspx?lang=eng, accessed 28 January 2017]). The CADORS are publicly-available incident reports, meant to provide information on aviation problems that involve: Canadian-registered aircraft, airports, airspace, or occurrences in international airspace for which Canada accepts responsibility. The data consist of reports from both people external to the UAV operations (e.g., other aviators, the public), and from UAV operators.

Reporting requirements for incidents vary considerably for UAV operators depending on operation type. Most recreational UAV users are not required to report problems. Commercial, civil, and recreational UAV operators certified by Transport Canada are generally required to report incidents according to conditions outlined in their Special Flight Operations Certificate (SFOC). Similarly, UAV operators flying under the exemptions in sections 602.41 and 603.66 of the Canadian Aviation Regulations (effective until 31 December 2019, 23:59 EST) are also required to report incidents to the nearest Transport Canada Civil Aviation office as outlined below:

a) Injuries to any person requiring medical attention.

b) Unintended contact between a UAV and persons, livestock, vehicles, vessels or other structures;

c) Unanticipated damage incurred to the airframe, control station, payload or command and control links that adversely affects the performance or flight characteristics of the UAV;

d) Anytime the UAV is not kept within the geographic boundaries and/or altitude limits as outlined in this exemption;

e) Any collision or risk of collision with another aircraft;

f) Anytime the UAV becomes uncontrollable, experiences a fly-away or is missing; and

g) Any other incident that results in a Canadian Aviation Daily Occurrence Report (CADORS).

These regulations have important implications for the type of incidents reported in the CADORS database. In particular, the reporting exemption for recreational operators severely constrains these data as self-reported incidents from recreational operators are generally missing. Other user reporting is primarily self-regulated; UAV operators are responsible for reporting themselves, with little oversight. These regulations are also subject to interpretation of the words 'unintended' and 'unanticipated'. Minor but common incidents (e.g., minor telemetry failures) could be considered as 'anticipated' by some operators. As a result, CADORS data cannot provide a complete picture of all incidents and safety concerns involving UAVs in Canada, but nevertheless do provide data on some concerns.

\subsection{Methods}

The CADORS database was searched for UAV incident reports by querying the keywords 'UAV,' 'UAS,' 'drone,' and 'unmanned', initially returning 169, 97, 162, and 190 reports, respectively. Another query was performed using the Aircraft Category field to return results classified in the database as 'Unmanned 
Aircraft'. The descriptions and/or narratives of each report were manually examined to remove identical and non-applicable results (e.g., "Springbank, AB Tower was unmanned briefly from 0320Z until 0330Z"). Five incidents involving UAVs and Canadian aircraft in US airspace were also removed. This resulted in 355 individual incident records involving UAVs from 05 Nov 2005 to 31 December 2016. Clear information regarding UAV size/weight was unavailable in most descriptions, thus all reports inclusive of the terminology above were considered in this analysis. We assume that most reports involve small UAVs due to the nature of the reports and the concurrent increase in report frequency with the wide availability of inexpensive systems, such as the DJI Phantom 1 which debuted in 2012. We manually classified reports based on the qualitative descriptions and quantitative distances detailed within the report narrative. These details were used to classify incident type using the following primary classification scheme:

Close encounter: Any report of a UAV flying within 500 feet (laterally or vertically) of another airspace user, or reports where pilots indicated evasive manoeuvres were required, or reports with the terms: 'near miss', 'close call', or similar.

Retroactive action: These are reports where action by Transport Canada was taken retroactively, usually from an online posting or retrieval of a crashed UAV.

Sighting: Incidents when one or more UAV(s) were sighted by pilot, public, or airport staff, but there was no reported close encounter with another airspace user.

Possible sighting: The sighting report contained some uncertainty as to whether the object was a UAV (e.g., balloon, bird, unidentified object).

Accident / minor incident: Accidents resulting in crash, property damage, and/or injury or minor incidents (e.g., misfiled paperwork, flying out of geographic boundaries).

We used the Aircraft Event Information field in the database to determine the number of incidents that reported an airspace infraction. The narratives were then used to extract more detailed information regarding the infraction, such as estimated altitude and proximity to aerodrome (if applicable). We examined these data to determine how many incidents occurred above the maximum altitude ceiling of 400 feet $(122 \mathrm{~m}$ ) above ground level (AGL) set by Transport Canada. For incidents with altitude specified above sea level (ASL), we estimated the altitude in AGL by measuring the ground elevation with Google Earth $^{\mathrm{TM}}$ in the vicinity of the incident or at the nearest aerodrome. The difference between the reported altitude (ASL) and the ground elevation provided and estimate of the UAV altitude (AGL). We assumed reports that did not specify altitude were in units ASL, and applied the same estimation procedure to calculate AGL values. Proximity to aerodromes was classified to be within, outside, or likely within (e.g., 'in the vicinity', 'on final approach', etc.) a radius of 5 nautical miles (nm).

Many of the reports in the CADORS database indicated UAV activity in airspace in which they were not likely to have clearance or certification. Therefore, we used this information to manually classify each report into groups based on the likelihood of airspace infraction using the following criteria:

Certain: The report was unambiguous in citing the lack of proper certification and/or clearance, such as an SFOC or Notice to Airmen/NOTAM (e.g., CADORS 2014P1479: "A NOTAM had been issued saying that the drone would be flying at an altitude no higher than $400 \mathrm{ft}$. AGL and not in the airport's immediate area.").

Probable: Insufficient details were presented, but the UAV was reported within $5 \mathrm{~nm}$ of an aerodrome, higher than 400 feet (122 m) AGL, flying at night-time, and/or above people. 
None: The narrative directly stated that the UAV was in compliance with regulations and within specifications (e.g., CADORS 2014P0646: "The UAV was being operated within all guidelines specified in the Special Flight Operating Certificate (SFOC) and the Open Permit application. At some point during the flight of the UAV, control was lost and it hit a building and fell to the street.").

Unknown: The report did not include enough detail to be classified into the above categories; most incidents placed into this class had no reported altitude or proximity to an aerodrome.

Additionally, we summarized the location (province) and time of occurrence (day-time or nighttime) attribute fields from the database and also used the narratives to determine any impact to operations, if the police were contacted, and, in the case of sighting incidents, who reported the sighting. Many reports were not sufficiently detailed to place in the above classes, and were marked as 'unknown'.

\subsection{Results}

\subsubsection{Incident type}

There were 355 UAV incidents in the CADORS database recorded between 05 Nov 2005 and 31 December 2016. Figures 2 and 3 summarize some of the key metrics described below. The majority of incidents were sightings $(n=236,66.5 \%)$. Sightings were followed by close encounters $(n=79,22.3 \%)$, accidents / minor incidents $(n=22,6.2 \%)$, possible sightings $(n=14,3.9 \%)$, and incidents involving retroactive action $(n=4,1.1 \%)$. The number of UAV sightings increased substantially after 2013 (Figure 2).

\subsubsection{Airspace infraction, altitude estimation, and proximity to aerodrome}

Airspace infractions were the common underlying trigger behind many of the incident reports. In total, 89.6\% ( $n=318)$ of all reports included a "Controlled airspace - unauthorized entry" designation. Based on the details included in the narratives, we independently determined 307 (86.5\%) probable airspace infractions, $18(5.1 \%)$ certain infractions, 8 (2.2\%) within legal limits, and 22 (6.2\%) unknown (Figure $3 b)$.

We identified 270 incidents $(76.1 \%)$ with reported estimates of the altitude at which the UAV activity occurred (primarily sightings and close encounters), or that had sufficient information to estimate the altitude using the ground elevation. Figure 4 shows a breakdown of the altitudes, revealing a decline in the number of incidents with increasing altitude. Though uncommon, it is concerning to note that several sightings and close encounters occurred above 10000 feet AGL. The data also indicate that 80.4\% of the 270 UAV incidents with reported or estimated altitude occurred above the standard maximum limit of 400 feet AGL (Figure 3c). Importantly, we estimate that at least $62.6 \%$ of the 270 incidents occurred at or above 1000 feet AGL, and at least $23.7 \%$ occurred above 3000 feet AGL.

Proximity to airports or aerodromes was also examined (Figure 3d). Specifically, 268 of the 355 reports $(75.5 \%)$ included an estimate of the distance from an aerodrome. Of these 268 reports, $54.8 \%(n=$ 147) occurred within $5 \mathrm{~nm}$ of an aerodrome, and 19.8\% $(n=53)$ likely occurred within this radius (e.g., "during the final approach..."). Of the 200 reports that occurred or likely occurred within $5 \mathrm{~nm}$ of an aerodrome, $145(72.5 \%)$ specify an altitude, of which $134(92.4 \%)$ were reported above the standard 100 feet ceiling within $5 \mathrm{~nm}$ of an aerodrome, and 111 (76.6\%) were above 300 feet.

Although the majority of impacts to operations were unknown or unspecified ( $n=167,47.0 \%)$, many reports specifically noted that there was no operational impact $(n=160,45.1 \%)$, while others cited some impact that we classified as ranging from minor to major (Figure 3e). Eleven reports (3.1\%) describe major impacts to operations. For example, one report described how a UAV grounded firefighting crews for 4 hours (CADORS 2015Q1884). Ten others described evasive manoeuvres by the piloted aircraft to avoid a UAV. There were also reports that indicated minor impacts $(n=17,4.8 \%)$, such as changing runway usage or flight patterns for short a duration (less than an hour). 
A host of additional details were extracted from the CADORS database and are summarized in Table 1 and Figure 3. Table 1 provides a provincial breakdown of incident reports. Figure 3 summarizes incident time of day (Figure 3f), whether police were contacted (Figure 3g), and source of reports (Figure $3 h)$.

\section{Discussion}

\subsection{CADORS reports and their source}

It is important to note that the CADORS system and similar incident reporting systems in the USA, such as the National Aeronautics and Space Administration (NASA) Aviation Safety Reporting System (ASRS), were developed with the intention of improving the safety of general aviation. The systems have recently been extended for reporting UAV incidents. This may need reconsideration. In our UAV incident data collected from the CADORS database, this reasoning is demonstrated by: (i) the limited perspective of these reports and (ii) the lack of clear details as they specifically relate to UAVs.

Most reports in our data were filed by pilots or other airport staff $(76.9 \%)$ or the public $(14.9 \%)$, with very few reports filed by UAV operators $(4.5 \%)$. The majority of sightings also came from pilots and/or airport staff (75.8\%). To some extent, this is unsurprising; these are the people most familiar with the CADORS and are directly affected by unsafe UAV operations. It is relevant to note that many of these sightings were likely outside legal airspace limits, and should have been reported by the UAV operator. However, it is probable that many similar incidents go unreported because they: (i) did not result in injury/property damage, (ii) were not observed by the public/other airspace user, or (iii) did not result in any other situation that involved the authorities. In general, incident reports from UAV operators are under-represented; these data do not provide a full picture of UAV safety in Canada.

Although the CADORS is not intended for direct enforcement of regulations, the system has, in some cases, been used by law enforcement agencies. As such, there is very little incentive for UAV operators to self-report incidents due to concern about fines, loss of capacity to operate a business, or concern about ambiguity in regulations. The CADORS is designed to improve aviation safety, not to punish, and without reports from UAV operators an understanding of incidents, accidents, and risks cannot be fully understood.

Data quality is a recurring problem for UAV incident reports. In the USA, the Federal Aviation Administration (FAA) and Department of Transportation recently released a public, online reporting system for reporting suspicious or illegal UAV activity; however, upon the debut of the system, they decided to revisit the design and subsequently withdrew the system (FAA, 2016). Transport Canada released a similar system at the end of 2016 to make it easier for Canadians to report unsafe drone operations (https://www.tc.gc.ca/eng/civilaviation/opssvs/drone-incident-report-form.html, accessed 28 January 2017). The intent of these reporting systems is understandable as they could provide an opportunity for UAV operators to report their incidents, but it also may result in large numbers of inaccurate or insufficiently detailed records from the public (especially as related to legality). The Academy of Model Aeronautics (an RC model aircraft organization) examined reports in the existing FAA database, which were predominantly from experienced pilots. Their results suggested that details within the reports can be unreliable and misleading (AMA, 2015). Expanding reporting to the general public would likely produce considerable amounts of unreliable data, unsuitable for basing policy.

\subsection{Close encounters and terminology}

Close encounters between commercial aircraft and UAVs represent a serious safety concern. Most commercial aircraft fly exclusively under Instrument Flight Rules (IFR) in controlled airspace where separation between aircraft is managed by air traffic control. Close encounters between commercial aircraft and UAVs suggest that: (i) the UAV was in controlled airspace, thus requiring an SFOC and 
NOTAM (which would specify communicating with air traffic control), and (ii) air traffic control did not know about the UAV.

Within the CADORS reports there is an attribute for Occurrence Category which has a classification for 'TCAS alert/Air Proximity/Loss of Separation /(Near) Mid-Air Collision' and an attribute for Aircraft Event in which the incident could be classified as 'Near Collision - Conflict' or 'Potential - Conflict'. Through our analysis using the Narrative field, these classifications from Transport Canada are not consistent in use. We define 'close encounter' similar to the definition of a near mid-air collision (NMAC) used by Joslin (2015) and adapted from the FAA Near Mid-Air Collision System (NMACS). The definition from NMACS is an aircraft coming within proximity of less than 500 feet of another aircraft, or a report of collision hazard between two aircraft from a flight crewmember or pilot.

Although the number of reports containing a TCAS alert/Air Proximity/Loss of Separation/(Near)

Mid-Air Collision designation in the CADORS was similar to our manual classification (86 and 79 , respectively), only 49 were common in both. Six reports that were considered a close encounter with the TCAS alert classification specified a larger distance between the UAV and manned aircraft than our defined 500 feet (e.g., "...came within 1 NM", “...1000 ft. above”). There were twenty-six reports that could not be classified as a close encounter by our criteria because they did not contain sufficient detail in the Narrative regarding UAV distance from the aircraft. There were also 30 occurrences that we manually classified as close encounters that were not considered such by the CADORS attributes. Of these reports, 4 specified the need for evasive piloting, 4 explicitly described a 'close encounter' or similar, and 8 reported a UAV proximity within 100 feet of a piloted aircraft (i.e. CADORS 2015O3394: "The flight crew reported a UAV passed approximately ten feet from their position"). Such incidents need to be clearly and consistently classified as close encounters by Transport Canada to improve understanding of UAV incidents.

\subsection{Airspace infractions and altitude estimates}

Airspace infractions were also classified by Transport Canada as part of the CADORS reporting. Almost all reports $(89.6 \%)$ in our dataset were classified as 'Controlled airspace - unauthorized entry'. This is commensurate with our classification (which did not consider this field). We found 325 (91.6\%) reports to be certain or probable airspace infractions. All of the reports classified by CADORS as a 'Controlled airspace - unauthorized entry', were found in the manual classification to be 'probable' or 'certain' airspace infractions, except for 10 that were classified as 'unknown' due to lack of details in the narrative, and 2 that were manually classified as 'none' because the narrative described that "the CASI (Civil Aviation Safety Inspector) was satisfied with the operator's information." This comparison demonstrates that the CADORS reports are reasonably reliable with respect to our classification of airspace infractions.

Many reports included unreliable UAV altitude estimates. Similarly, many reports did not distinguish between altitude in AGL and elevation in ASL. We examined each narrative to reduce the ambiguity. Even with some uncertainty, the majority of sightings $(80.4 \%)$ would still be far above the standard 400 feet AGL maximum operating ceiling for UAVs, with 62.6\% above 1000 feet AGL, 24.1\% above 3000 feet AGL (Figure 3).

Gettinger and Michel (2015) performed a similar analysis of reported incidents involving UAVs and piloted aircraft in the US National Airspace System (NAS). They compiled 921 records of incidents involving UAVs and piloted aircraft in the 21-month period between 17 December 2013 and 12 September 2015. Using a simple classification scheme (only concerned with sightings and close encounters), they found $64.5 \%$ of the incidents were sightings, while $35.5 \%$ were close encounters. The total number of sightings and close encounters from the CADORS database in the same period was 113, of which $61.9 \%$ were sightings or potential sightings, $31.0 \%$ were close encounters, and $7.1 \%$ were UAV accidents / minor incidents (such as misfiled paperwork). We note that 7 of the potential sightings included some uncertainty if the object spotted was a UAV or a balloon.

Of the reports in the US, Gettinger and Michel (2015) found that more than 90\% described UAVs above 400 feet, the maximum altitude allowable for UAV operations in the USA. This is higher than our results from the 270 CADORS reports that included altitudes, in which $80.4 \%$ were reported above 400 
feet. The lower number in Canada may be due to higher compliance with regulations by UAV operators; however, it must also be noted that the standard legal altitude in Canada is 300 feet AGL if flying under any exemption or holding a Simplified UAV Operator SFOC (400 feet AGL if an operator holds a Complex UAV Operator SFOC). From the 270 CADORS reports that contain altitude estimates, $85.4 \%$ were reported at or above the standard 300 feet limit, suggesting a substantial number of non-compliant UAV operations in Canada.

Some of the most sensitive and at risk airspace is near aerodromes, because of concentrated aviation traffic. Regulations limit the operation of UAVs within a distance of $5 \mathrm{~nm}$ from the centre of any aerodrome. Gettinger and Michel (2015) found that 72.2\% of UAV incident reports in the USA included information about distance to the nearest aerodrome. This is fairly consistent with the $75.5 \%$ of reports from the CADORS database that specify a distance. From the US reports they discovered that $58.8 \%$ were within $5 \mathrm{~nm}$ of an aerodrome, which is much lower than the $74.6 \%$ we found to be within or likely within $5 \mathrm{~nm}$ of an aerodrome in the CADORS reports. These data suggest a higher fraction of incidents in Canada are occurring close to aerodromes than in the United States. Not only are a notable number occurring near aerodromes, but $92.4 \%$ of incidents that occurred within $5 \mathrm{~nm}$ of an aerodrome specified an altitude above the maximum legal altitude of 100 feet AGL. Additionally, $76.6 \%$ were flying above 300 feet AGL.

\subsection{Potential solutions}

The regulations surrounding small UAV operations in Canada are clear and widely accessible online. However, the CADORS data suggest that the regulations are not being followed. A lack of compliance could be the result of an: (i) absence of UAV operator knowledge, (ii) inadvertent loss of control, or (iii) intended defiance and disregard for the regulations. Each of these issues requires a different approach (see also Clarke and Moses, 2014).

A lack of UAV operator knowledge is plausibly seen as the most prevalent issue given the wide availability and low cost of UAVs. Although UAV manufacturers and retailers include operation manuals, instructions, and legal warnings - it is unlikely that the novice operator will fully comprehend airspace rules and the risks of unsafe operations. This lack of aeronautical knowledge is a serious risk and has been acknowledged by various governing bodies, aviation associations, and UAV manufacturers. For example, Transport Canada provides an abbreviated and easy to understand "Do's and Don'ts" for flying a UAV safely and legally (see: http://www.tc.gc.ca/eng/civilaviation/opssvs/flying-drone-safelylegally.html, accessed 28 January 2017). This effort provides some of the most pertinent safe flying information from the lengthy UAV legislation in a single, easy to understand format. Similar, but more mature efforts are widespread in the USA. The FAA has created a mobile app for users to understand what type of airspace they are flying in and regulations associated with that airspace. This is much simpler than a traditional approach. For example, consulting an aeronautical chart requires a UAV operator to: (i) purchase a chart, (ii) have the chart on hand when considering flying, and (iii) have the skills to understand the complex notation on the chart. Part of increasing operator knowledge is decreasing the barriers to knowledge uptake; it should be easy for users to understand the regulations. More campaigns and innovative methods, such as these, for disseminating knowledge to new UAV operators will likely increase UAV and aviation safety.

Inadvertent loss of control of UAVs may also result in an airspace infraction and/or present a risk to other airspace users. In our CADORS dataset, 6 reports indicated loss of control out of the 16 reports from UAV operators. Although technological advances will certainly increase UAV reliability, appropriate protocols are required to manage these issues. This is an extension of increasing UAV operator knowledge as operators need to understand the limitations of their equipment, including all potential causes of communication failure (e.g. radio interference, electromagnetic field disturbances, transmission obstacles, or failure of components). Most of these issues can be mitigated through UAV operator awareness of the flight area and proper planning. For example, CADORS 2016P0859, in which the UAV operator lost radio link and control of the UAV while going into a failsafe mode because it 
manoeuvred behind a hill, could have been prevented by planning proper failsafe manoeuvres to keep the UAV away from obstacles and within communication of the ground station.

More importantly, however, Transport Canada should lay out clear protocols (as they have with the "Do's and Don'ts) for when and who to contact in regards to a loss of control/communication. The lack of clarity in the protocol is evident through the CADORS database in which some of these incidents were directed through the nearest Flight Service Station (FSS) or the Flight Information Center (FIC), while others came from the local police. These reports are essential for the safety of piloted aircraft and need to be sent through the proper channels of communication in a timely manner to ensure the safety of all other aircraft in the area.

Intentional disregard for the regulations is much more complicated to address. This requires enforcement of existing regulations. Many of the CADORS reports discussed follow up action by police or security (44.2\%), but often the operator of the UAV could not be found, particularly in urban areas. This makes enforcement of illegal and unsafe UAV operations difficult. The FAA has moved towards requiring registration of all UAVs (including recreational and non-recreational UAVs), which allows the ability of tracking a UAV back to the owner, even if the owner cannot be found on site. However, in the case of the CADORS data, most incidents are mid-air sightings in which it is very difficult to distinguish a registration number. While it is unclear how many penalties for unsafe behaviour can be levied - these types of efforts can act as deterrents. It is important to note that these efforts are aimed at safety, rather than security or privacy concerns (see other analyses: Bracken-Roche et al., 2014; Wang et al., 2016).

Although regulating UAV operators is seen as the first approach to improving safety - this neglects the unique characteristic of UAVs of being completely computer controlled and often connected to the internet. Commands from the user, or an autonomous navigation system, are interpreted by the flight computer. The flight computer can be used as an evaluation layer, stopping the user from executing commands that are in contravention of regulations. Such an evaluation layer is not possible or acceptable in piloted aircraft, where the pilot is understood to have the final say in actions. Several manufacturers of popular commercial UAVs, such as DJI, 3D Robotics (3DR), and Yuneec, have started implementing hardware/software solutions that utilize the flight computer and GPS to prevent potentially illegal flights.

Geofencing is one of these emerging methods designed to stop UAVs from flying in restricted airspace. Geofencing is seen as a viable alternative to regulating users, and has seen wide adoption in commercial UAVs as a preventative measure (e.g., "DJI Geo system"). It is important to note that geofencing is not (currently) a regulatory requirement. Nor is geofencing a perfect solution - as it invariably can be circumvented by motivated individuals - but it is likely to stop the majority of small UAV users from unknowingly committing airspace infractions.

\section{Conclusions}

UAVs are a growing component of Canadian airspace and are set to benefit from vast investment in the next decade estimated around $\$ 1.8$ billion in Canada alone (Strategic Defence Intelligence, 2013). However, ensuring safety is an important concern that requires a deep understanding of UAV issues. Given that the small UAV industry is nascent and rapidly evolving, there is an imperative to set clear safety guidelines and standards with the aims to both improve current operations and motivate the development of new safety technologies.

To gain some insight into the types of incidents that are presently occurring with small UAVs we analyzed the Transport Canada CADORS database of civilian aviation incidents. While these data are constrained by reporting criteria, they do highlight some safety concerns and reveal some important trends of UAV incidents within Canada. The majority of reported incidents were sightings and close encounters with piloted aircraft, which often represented probable or certain airspace infractions. The number of sightings dramatically increased after 2013, suggesting the problem of airspace infractions is real and increasing in prevalence. UAVs operating in controlled airspace without appropriate permissions not only violate the regulations, but also pose a serious risk to piloted aircraft, as demonstrated by the rise in close encounters. 
We discuss some of the inherent and potential causes of airspace infractions and review emerging techniques that may provide solutions for reducing such violations. The UAV incident data suggest that changes to regulations, education, and/or enforcement is warranted. The suite of communications and public education campaigns by Transport Canada in2016 did not reduce the number of near misses and airspace infractions; instead, they increased. As discussed here, blanket adaptation of manned aviation regulations to small UAV safety is often only partially effective, and does not take advantage of the unique characteristics of UAVs. We hope this synthesis provides basing for more effective future policy.

\section{Acknowledgements}

We gratefully acknowledge project funding from the University of Calgary. We thank Stephen Myshak, Allison Cully, Jeff Boyd, Dave Curry, and Simon Brink for helpful discussion. We also thank two anonymous reviewers for providing feedback that has improved this paper

\section{References}

AMA: Academy of Model Aeronautics. 2015. A Closer Look at the FAA's Drone Data [Press Release]. Retrieved from https://www.modelaircraft.org/gov/docs/AMAAnalysis-Closer-Look-at-FAA-DroneData_091415.pdf [last accessed 28 January 2017].

Bracken-Roche, C., Lyon, D., Mansour, M.J., Molnar, A., Saulnier, A., and Thompson, S. 2014. Surveillance drones: Privacy implications of the spread of unmanned aerial vehicles (UAVs) in Canada. Quebec: Surveillance Studies Centre.

Clarke, R., and Moses, L.B. 2014. The regulation of civilian drones' impacts on public safety. Computer Law \& Security Review 30: 263-285. doi: 10.1016/j.clsr.2014.03.007.

Clothier, R.A., Greer, D.A., Greer, D.G., and Mehta, A.M. 2015. Risk perception and public acceptance of drones. Risk Analysis 35: 1167-1183. doi: 10.1111/risa.12330.

Clothier, R.A., Williams, B.P., Coyne, J., Wade, M., and Washington, A. 2015a. Challenges to the development of an airworthiness regulatory framework for unmanned aircraft systems. In $16^{\text {th }}$ Australian Aerospace Congress, 23-24 February 2015, Melbourne, Australia. pp.87-98.

FAA: Federal Aviation Administration. 2016. Federal Register 81 FR 30600. Retrieved from https://www.federalregister.gov/documents/2016/05/17/2016-11573/agency-information-collectionactivities-requests-for-comments-clearance-of-renewed-approval-of [last accessed 28 January 2017]

Gettinger, D., and Michel, A.H. 2015. Drone sightings and close encounters: An analysis. Bard College Center for the Study of the Drone.

Joslin, R. 2015. Synthesis of Unmanned Aircraft Systems Safety Reports. Journal of Aviation Technology Engineering 5: 2-6.

Hugenholtz, C.H., Moorman, B.J., Riddel, K., and Whitehead, K. 2012. Small unmanned aircraft systems for remote sensing and earth science research. Eos, Transactions American Geophysical Union, 93(25), 236-236.

Hugenholtz, C.H., Walker, J., Brown, O., Myshak, S. 2015. Earthwork volumetrics with a UAV and softcopy photogrammetry. Journal of Survey Engineering 141(1), 06014003.

Manning, S.D., Rash, C.E., LeDuc, P.A., Noback, R.K., and McKeon, J. 2004. The Role of Human Causal Factors in U.S. Army Unmanned Aerial Vehicle Accidents. USAARL Report No. 2004-11.

Merat, N., Jamson, A.H., Lai, F.C.H., Daly, M., and Carsten, O.M.J. 2014. Transition to manual: driver behaviour when resuming control from a highly automated vehicle. Transportation Research Part F 27: 274-282. doi: 10.1016/j.trf.2014.09.005.

Stevenson, J.D., O'Young S., and Rolland, L. 2015. Estimated levels of safety for small unmanned aerial vehicles and risk mitigation strategies. Journal of Unmanned Vehicle Systems 3: 205-221. doi: 10.1139/juvs-2014-0016.

Strategic Defence Intelligence. 2013. The UAV Market in Canada to 2023: Market Brief. DF2403MB. $15 \mathrm{p}$.

Tamminga, A., Eaton, B., and Hugenholtz, C.H. 2015. UAS-based remote sensing of fluvial change following an extreme flood event. Earth Surface Processes \& Landforms 41(11), 1464-1476. 
Wang, Y., Xia, H., Yao, Y., and Huang, Y. 2016. Flying eyes and hidden controllers: A qualitative study of people's privacy perceptions of civilian drones in the US. Proceedings on Privacy Enhancing Technologies 3: 172-190. doi: 10.1515/popets-2016-0022.

Whitehead, K., Hugenholtz, C.H., Myshak S., Brown, O., LeClair, A., Tamminga, A., Barchyn, T.E., Moorman, B., and Eaton, B. 2014. Remote sensing of the environment with small unmanned aircraft systems (UASs), part 2: scientific and commercial applications. Journal of Unmanned Vehicle Systems 2: 86-102. 
Figure 1
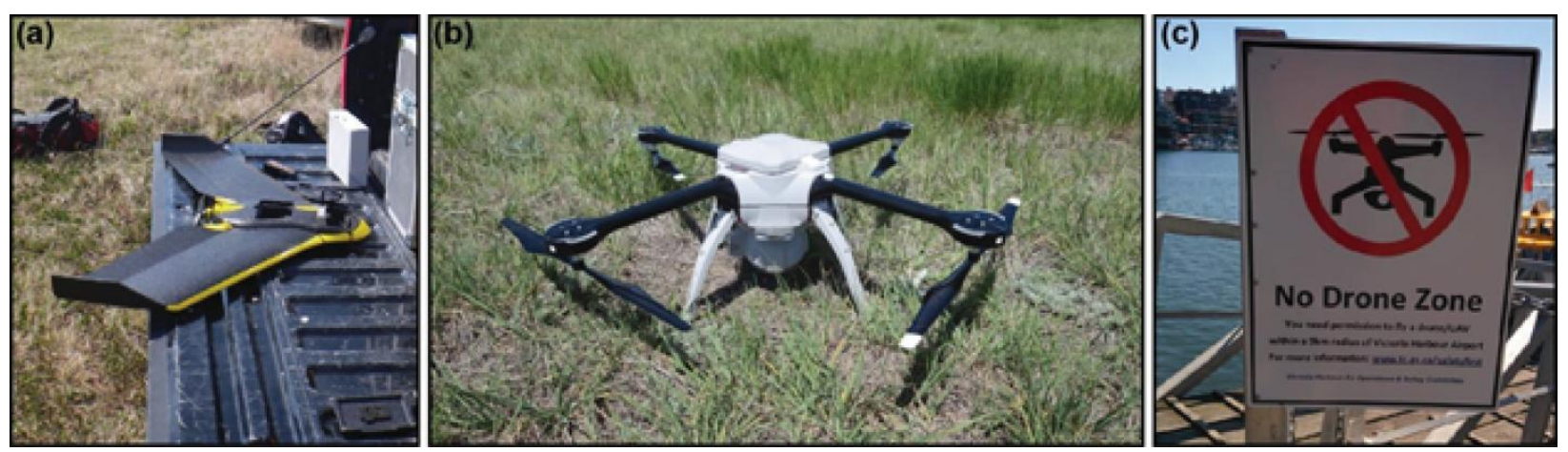
Figure 2

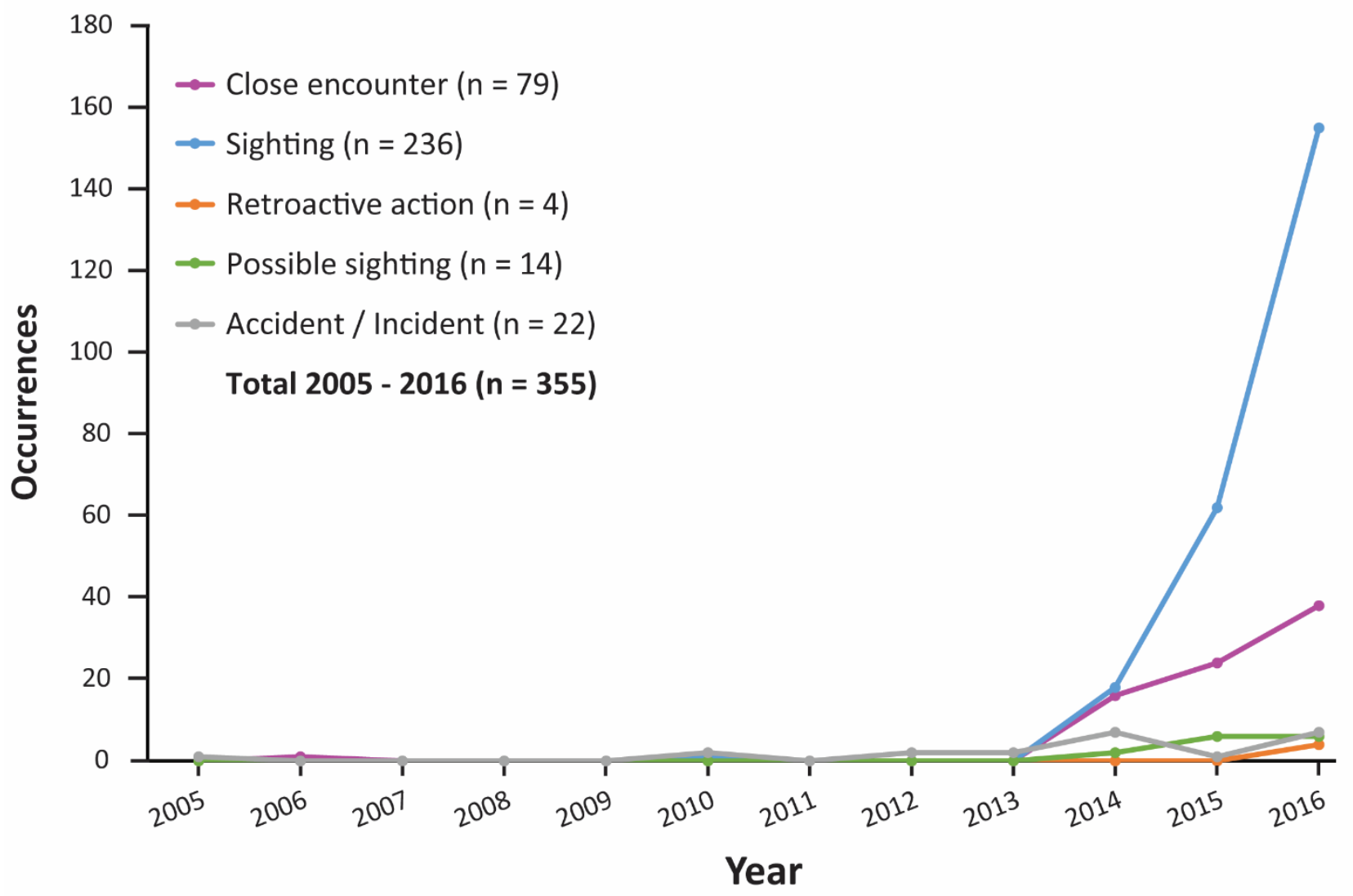


Figure 3

(a) Incident type

( $n=355$ )

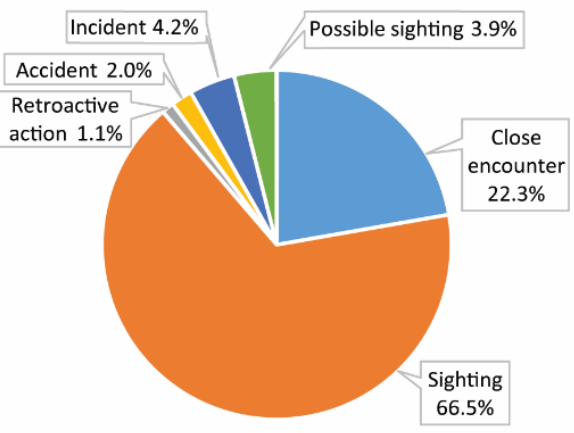

(b) Probability of airspace infraction ( $n=355)$

(c) UAV altitude ( $n=270$ )

(d) UAV within $5 \mathrm{~nm}$ of an aerodrome ( $n=268$ )

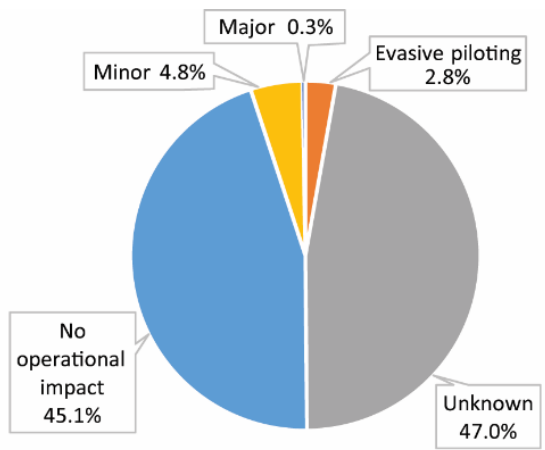

(e) Operational impact ( $n=355$ )

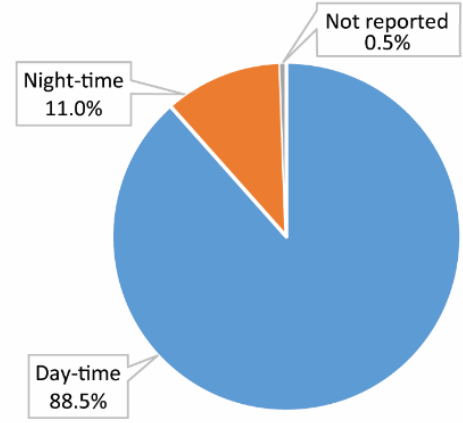

(f) Time of occurrence ( $n=355$ )

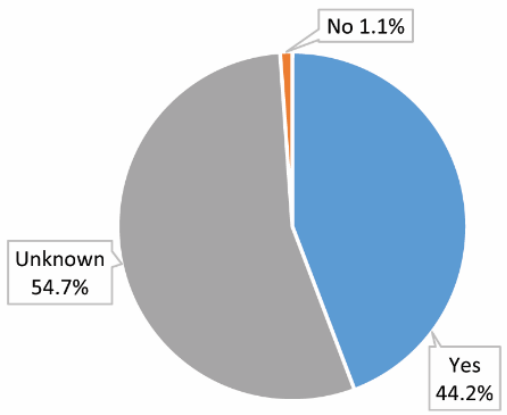

(g) Police contacted? ( $n=355$ )

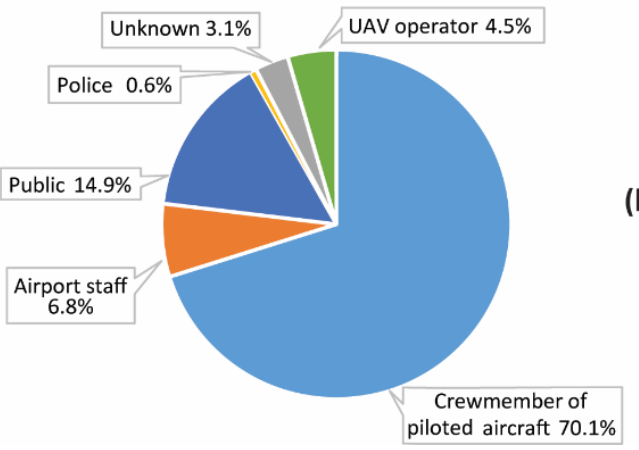

(h) Source of report ( $n=355$ ) 
Figure 4

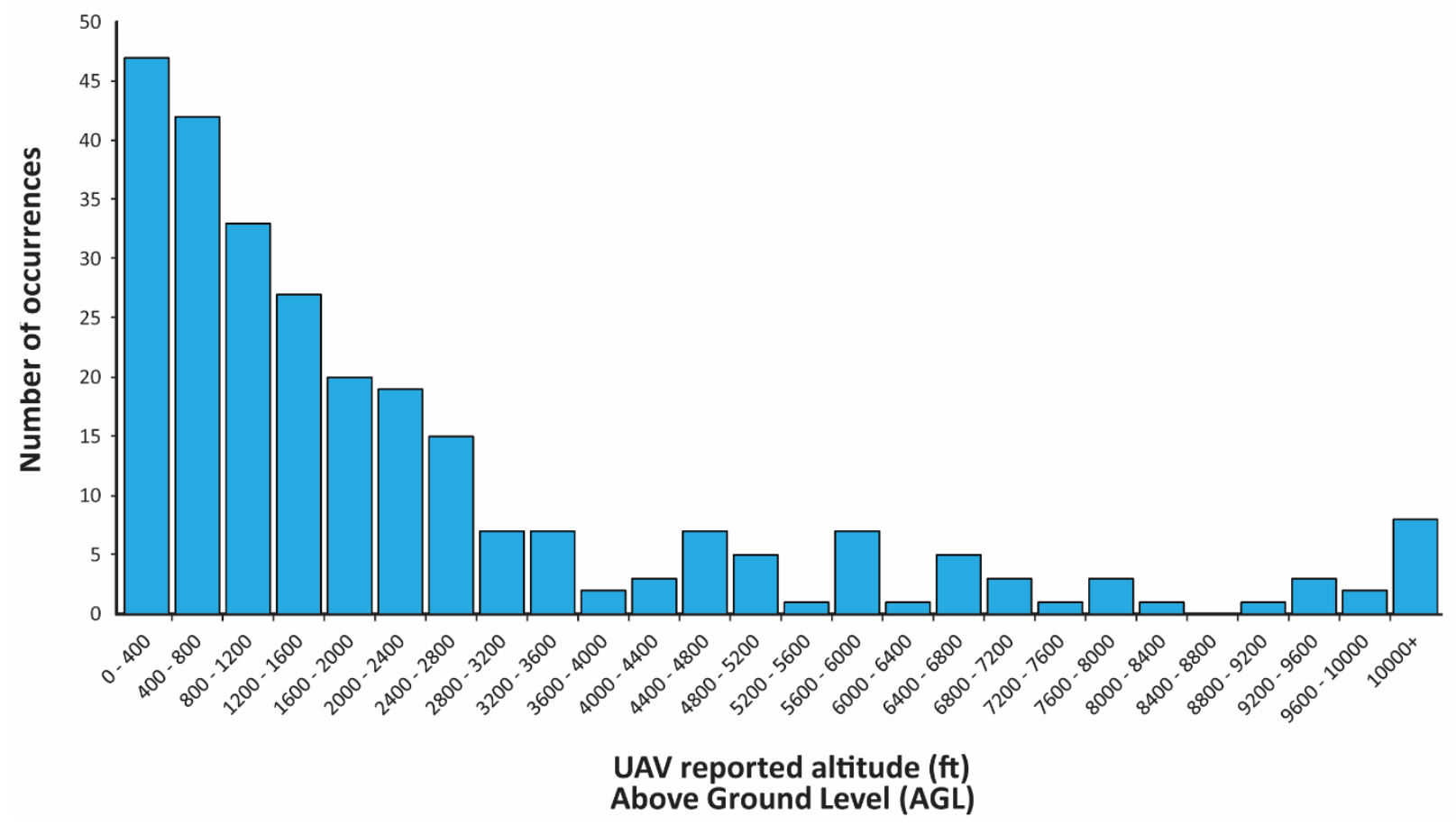


Table 1

\begin{tabular}{|c|c|c|c|c|c|c|}
\hline Jurisdiction & Sighting & $\begin{array}{c}\text { Close } \\
\text { encounter }\end{array}$ & $\begin{array}{c}\text { Retroactive } \\
\text { action }\end{array}$ & $\begin{array}{c}\text { Accident/ } \\
\text { incident }\end{array}$ & $\begin{array}{c}\text { Possible } \\
\text { sighting }\end{array}$ & $\begin{array}{c}\text { Total UAV } \\
\text { reports per } \\
\text { province }\end{array}$ \\
\hline BC & 83 & 25 & 2 & 3 & 3 & 116 \\
\hline AB & 18 & 8 & 0 & 2 & 1 & 29 \\
\hline SK & 3 & 3 & 0 & 4 & 1 & 11 \\
\hline MB & 9 & 5 & 0 & 0 & 1 & 15 \\
\hline ON & 80 & 21 & 1 & 6 & 6 & 114 \\
\hline QC & 32 & 11 & 1 & 2 & 1 & 47 \\
\hline NB & 3 & 0 & 0 & 0 & 0 & 3 \\
\hline NS & 1 & 1 & 0 & 2 & 1 & 5 \\
\hline PE & 1 & 0 & 0 & 0 & 0 & 1 \\
\hline NL & 2 & 5 & 0 & 1 & 0 & 8 \\
\hline YK & 2 & 0 & 0 & 0 & 0 & 2 \\
\hline NU & 1 & 0 & 0 & 0 & 0 & 1 \\
\hline NW & 1 & 0 & 0 & 2 & 0 & 3 \\
\hline
\end{tabular}




\section{Figure Captions:}

Figure 1. UAVs with wide commercial use fall generally into (a) fixed wing, or (b) and rotary wing models, and although less common, there are hybrid types. (c) Signs adjacent to airports have been installed in some locations to deter UAV operators from illegally flying in restricted airspace; this example is from Victoria Harbour Airport, Victoria, British Columbia, Canada.

Figure 2. Classification of different incidents over time. Note that this was prepared with data from 05 November 2005 to 31 December 2016.

Figure 3. Breakdown of UAV incident metrics. The number $(n)$ of incident reports for each metric are listed in brackets.

Figure 4. Reported or estimated altitudes (AGL) of UAV incidents from the CADORS database, 05 November 2005 to 31 December 2016.

\section{Table Caption:}

Table 1. CADORS UAV incident reports by province. 